\section{Acute jejunal gangrene with perforation due to Ascaris lumbricoides}

\section{Introduction}

Infestation with Ascaris lumbricoides is a worldwide phenomenon. While most patients with Ascaris infection are asymptomatic, life threatening gastrointestinal conditions may develop that require immediate medical and surgical intervention. ${ }^{1}$ This report documents a case of perforation with gangrene of the intestine in a 25 year old male.

\section{Case report}

A 25-year-old male presented to the emergency with three day history of epigastric pain associated with nausea, anorexia and vomiting. The patient was febrile and tachypneic on examination. Abdominal examination revealed a hugely distended abdomen and board-like rigidity on palpation. There was generalized tenderness with rebound tenderness. Bowel sounds were absent.

An abdominal radiograph taken in the supine and erect positions showed air under the diaphragm with several airfluid interfaces and free air in the abdomen. With a clinical diagnosis of acute abdomen due to hollow viscus perforation, an exploratory laparotomy was performed. Per-operatively, distal jejunum was found gangrenous with multiple perforations. Resection and anastomosis was performed and

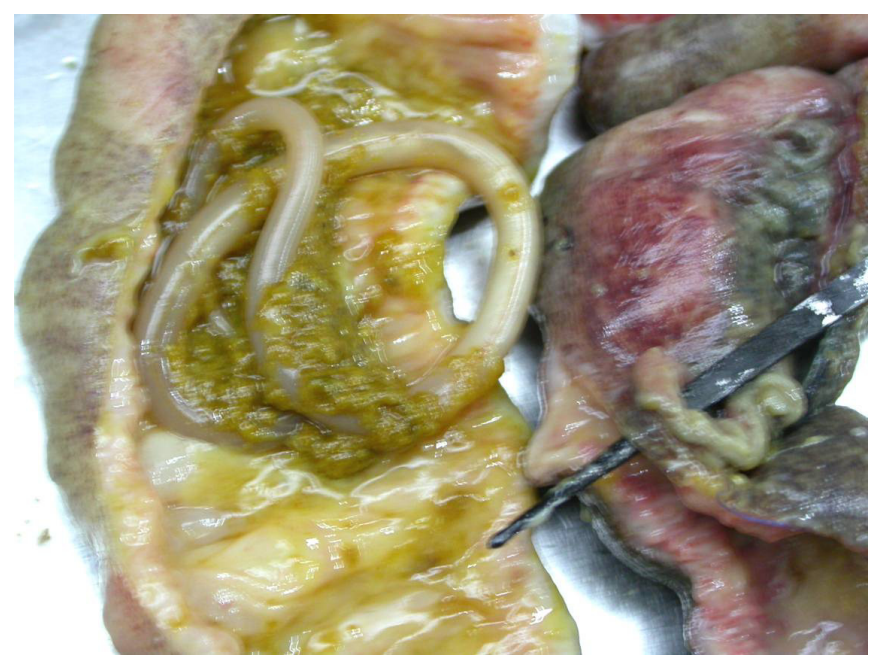

Figure 1: Single live Ascaris worm obstructing the lumen resulting in multiple perforations of small intestine (probe through one of the perforation) specimen was sent for histopathology. Gross examination showed serosal exudates with ulceration of mucosa and an Ascaris worm in the lumen (Figure 1). Microscopy showed mucosal gangrene with submucosal inflammatory infiltrate. A final diagnosis of jejunal gangrene with perforation due to ascariasis was made. Post-operatively, the patient received a course of mebendazole and is asymptomatic over one year follow-up period.

\section{Discussion}

The commonest complication of ascariasis is intestinal obstruction due to a worm bolus. ${ }^{2}$ The obstruction may be acute or subacute. It can be complicated by intussusception, perforation and gangrene of the bowel. In ascariasis, the cause of perforation of the small intestine remains controversial, with two main theories. In the tropics patients consistently have histories of diseases associated with ulceration of the intestines such as typhoid enteritis, tuberculosis and amebiasis. During extreme conditions, such as inflammation, starvation or worm bolus obstruction, some parasites are believed to migrate into the ulcers and cause perforations. ${ }^{2}$ Another possible explanation is that the large worm bolus can lead to pressure necrosis and gangrene. ${ }^{3}$ The diseased bowel thus becomes susceptible to rupture by the burrowing action of the worm. ${ }^{4}$ Intestinal obstruction and gangrene constitute the most common surgical complications of ascariasis. The mechanism of obstruction is occlusion of the intestinal lumen by worms packed in the distal ileum as well as localized volvulus of a segment of terminal ileum owing to the weight of the roundworms inside. The mechanism of gangrene may be due to pressure necrosis caused by the roundworms and the accompanying localized volvulus. In patients with ascariasis who present with acute surgical conditions, an urgent laparotomy is indicated to deal with intestinal perforation, to control peritonitis and to decompress the worm bolus that may be causing the acute intestinal obstruction. ${ }^{1}$ In endemic areas, mass treatment with mebendazole should be advocated so as to reduce morbidity and although ascariasis is very rare in developed countries, yet clinicians should consider Ascaris infection in the differential diagnosis of acute abdomen, due to current age ease of traveling, and acquired infections in travelers to tropical regions.
NIDHI GOYAL NITA KHURANA 


\section{Correspondence: Dr. Nidhi Goyal Department of Pathology, Maulana Azad Medical College \\ New Delhi, India \\ Email:goyalnidhi@rediffmail.com}

\section{References}

1. Sridhar VB, Peter JH: Acute Gl and Surgical complications of Ascaris lumbricoides infection. Infect Med 2003; 20:154-9

2. Efem SE. Ascaris lumbricoides and intestinal perforation. $\mathrm{Br} J$ Surg. 1987;74:643-4.

3. Surendran N, Paulose MO. Intestinal complications of round worms in children. J Pediatr Surg.1988;23:931-5.

4. Khuroo MS. Ascariasis. Gastroenterol Clin North Am. 1996;25:553-77.

\section{Multicentric biliary papillomatosis with synchronous gallbladder malignancy}

\section{Introduction}

Adenomas and papillomas of gallbladder are quite uncommon. We present here a case of a 54-year-old female with gallbladder papillomatosis.

\section{Case report}

A 54 year old patient presented with the history of a right upper quadrant mass and typical biliary symptoms. Abdominal examination revealed a firm globular mass in the right hypochondrium, consistent with a mass originating from the gallbladder. The patient's hemoglobin was $12.6 \mathrm{~g} / \mathrm{dl}$, and WBC count was $8,500 / \mathrm{mm}^{3}$ (neutrophil $76 \%$, lymphocyte $21 \%$, and eosinophil 3\%). Carcinoembryonic antigen and CA 19-9 were normal. Chest X-ray was normal. Pre-operative diagnosis was suggested by USG abdomen and CECT which showed a gallbladder mass. FNAC reported it to be an adenocarcinoma. A radical cholecystectomy with CBD excision and Roux-en-Y hepaticojejunostomy was performed. Gross description and microscopic examination confirmed the diagnosis of benign diffuse papillomatosis involving the entire mucosal surface of the gallbladder, cystic duct and bile duct. Histopathology revealed multicentric papillomatosis with in-situ

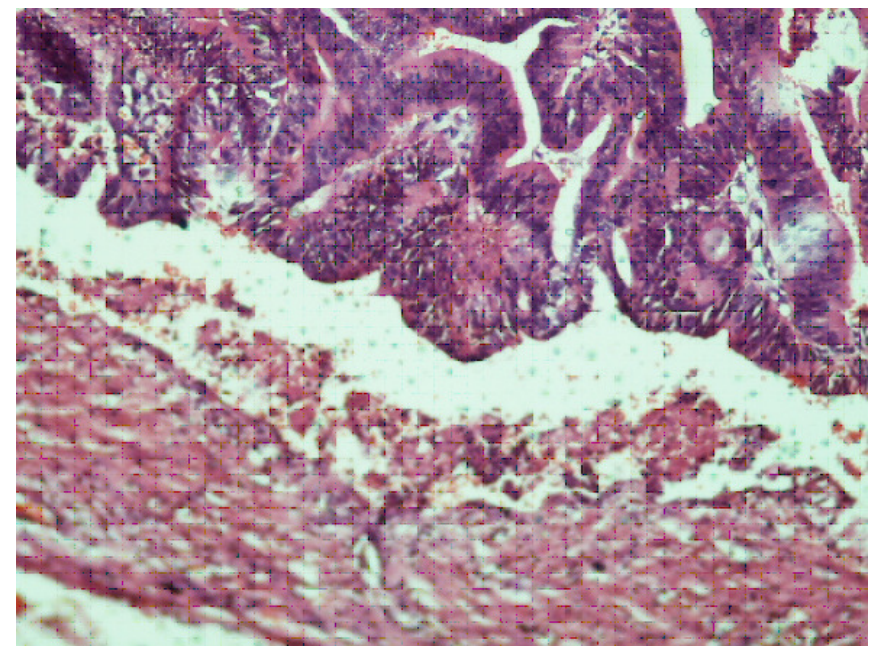

Figure 1a. H \& E section of gallbladder showing polypoidal projections, mucin and in situ carcinoma

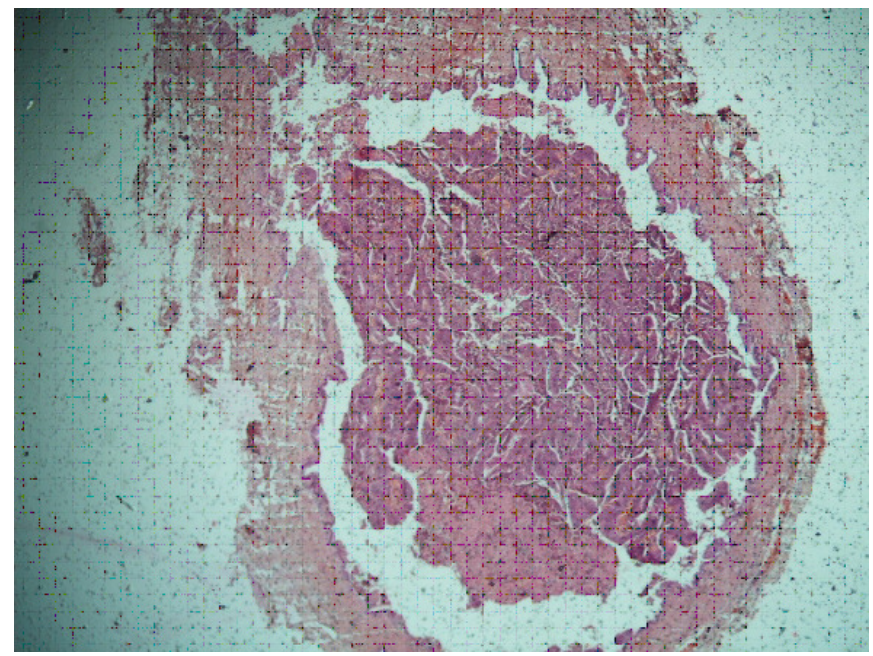

Figure 1b. H \&E section of bile duct showing polyps and mucin

adenocarcinoma of the gallbladder (Stage T1a) (Figure 1a $\boldsymbol{\&}$ b).

\section{Discussion}

Polypoid preinvasive lesions of the biliary tract have been termed "adenomas", "papillomas", "papillomatosis (adenomatosis)", and "papillary adenocarcinomas", based on their growth pattern, multiplicity, extent and degree of malignant change. ${ }^{1-3}$ Conceptually, these lesions represent the biliary counterpart of colonic adenomas and follow a similar adenomacarcinoma sequence. Some of the clinical and pathologic characteristics of these lesions are similar to pancreatic intraductal papillary mucinous neoplasms, which has led some authors to refer to these tumors as "biliary intraductal papillary mucinous neoplasms". ${ }^{1}$ Adenomas occur predominantly in 